Relations industrielles

Industrial Relations

\title{
Towers, Brian, The Representation Gap: Change and Reform in the British and American Workplace
}

\section{Michel Brossard}

Volume 54, numéro 3, 1999

URI : https://id.erudit.org/iderudit/051257ar

DOI : https://doi.org/10.7202/051257ar

Aller au sommaire du numéro

Éditeur(s)

Département des relations industrielles de l'Université Laval

ISSN

0034-379X (imprimé)

1703-8138 (numérique)

Découvrir la revue

Citer ce compte rendu

Brossard, M. (1999). Compte rendu de [Towers, Brian, The Representation Gap: Change and Reform in the British and American Workplace]. Relations industrielles / Industrial Relations, 54(3), 613-615.

https://doi.org/10.7202/051257ar

Tous droits réservés @ C Département des relations industrielles de l'Université Laval, 1999
Ce document est protégé par la loi sur le droit d'auteur. L'utilisation des services d'Érudit (y compris la reproduction) est assujettie à sa politique d'utilisation que vous pouvez consulter en ligne.

https://apropos.erudit.org/fr/usagers/politique-dutilisation/ 


\title{
Recensions
}

\section{Book Reviews}

\author{
The Representation Gap: Change and Reform in the British and American \\ Workplace \\ par Brian TOWERS, Oxford: Oxford University Press, 1997, 294 p., ISBN 0-19- \\ 829319-4.
}

Comment interpréter la baisse de la représentation syndicale et l'affaiblissement de la négociation collective? Devant l'évolution du phénomène, des mécanismes supplémentaires de représentation devraient-ils être prévus pour assurer l'exercice de la démocratie industrielle dans les milieux de travail ? C'est à ces deux questions principales que cherche à répondre cet ouvrage en comparant l'Angleterre et les États-Unis.

Les quatre premiers chapitres étudient l'affaiblissement de la représentation des salariés et présentent son interprétation. L'analyse est structurée exclusivement autour du recul du syndicalisme qui constitue dans ces deux pays le seul mécanisme institutionnalisé de la défense indépendante des intérêts des salariés. La tendance observable en Angleterre depuis 1979 et aux États-Unis, depuis les années soixante débouche avec des taux respectifs de syndicalisation de $33 \%$ et $15 \%$ en 1995 sur ce que l'auteur considère comme une véritable crise de la représentation ouvrière et de ses effets sur les conditions de travail. Cette crise apparaît à peu près inéluctable en vertu de l'évolution des facteurs structurels propres aux sociétés industrialisées et d'autres, plus spécifiques à chacun des pays étudiés.

Le modèle fonctionne à partir d'une mécanique assez simple : l'évolution des facteurs structurels a tendance à entraîner un recul de la représentation dont le mouvement peut-être soit amplifié, soit freiné, soit contrecarré par celle des autres facteurs et plus spécialement parmi eux le degré d'opposition des employeurs et le contenu des politiques publiques. La prévision de l'évolution de ces deux derniers facteurs rend d'ailleurs l'auteur plus optimiste envers la situation de l'Angleterre. Aux États-Unis, le contexte plus défavorable exigera que les syndicats misent beaucoup plus sur euxmêmes pour redresser la situation. Dans les deux pays étudiés, les syndicats ont d'ailleurs pris des mesures internes (augmentation des cotisations ; fusion de syndicats...) et externes (nouvelle approche de recrutement ; collaboration avec l'employeur...) pour faire face au phénomène. Même si l'auteur reconnaît que les syndicats ne peuvent exercer par euxmêmes qu'une influence somme toute très faible sur le recul de la représentation, il est d'avis que pour être efficace leur action doit être axée en priorité sur le milieu de travail.

Cette baisse de la présence syndicale entraîne une crise de la représentation des salariés dans chacun des deux pays. De plus, peu d'indices permettent de prévoir un avenir immédiat plus encourageant même si le cas anglais suscite plus d'optimisme que le cas américain. Que faire alors? C'est cette question qui fait l'objet des quatre derniers chapitres.

Le chapitre cinq présente la thèse de l'ouvrage. La démocratie industrielle 
dans l'entreprise doit être définie comme le prolongement de la démocratie politique dans la société et être, à ce titre, considérée comme essentielle. Ce principe est absolu et ne doit pas dépendre de ses implications économiques. Pour assurer l'exercice de la démocratie industrielle, les moyens utilisés doivent permettre la représentation indépendante des intérêts des salariés. Le syndicalisme et deux autres mécanismes (le partenariat patronal-syndical et les comités d'entreprise de type allemand) correspondent à cette exigence. Plusieurs pratiques innovatrices introduites dans les entreprises au cours de la dernière décennie sont exclues parce qu'elles poursuivent prioritairement les objectifs des employeurs, parfois au détriment des syndicats et de leurs membres. La collaboration patronale-syndicale est visée plus particulièrement parce qu'elle rend difficile pour les syndicats la défense indépendante des intérêts, des valeurs et des droits des salariés. Les chapitres six et sept analysent successivement l'évolution de la démocratie industrielle au niveau des milieux de travail dans chacun des deux pays. Malgré quelques changements et l'espoir suscité aux États-Unis par la création de la Commission Dunlop en mars 1993, l'auteur conclut que la représentation indépendante des intérêts des salariés au sein des entreprises a connu une érosion continue de part et d'autre de l'Atlantique au cours des deux dernières décennies.

Le recul de la représentation a été compensé partiellement par le renforcement de la régulation légale. Toutefois cette protection axée beaucoup plus sur les droits individuels que collectifs n'est pas aussi flexible que celle procurée par une convention collective sans compter qu'elle est aussi plus difficile à faire respecter. C'est pourquoi le chapitre huit se penche sur la possibilité d'introduire dans les entreprises américaines et anglaises deux autres formes de démocratie industrielle: les conseils d'entreprises inspirés du cas allemand (Betriebsrat) et le partenariat patronal-syndical.
En ce qui concerne les conseils d'entreprise, l'expérience allemande montre qu'ils peuvent très bien être utilisés comme des compléments à la négociation collective et coexister de façon harmonieuse avec l'organisation syndicale. Toutefois le contexte américain apparaît peu propice à leur introduction. Non seulement le contexte politique (le contrôle par les républicains de la Chambre des Représentants et du Sénat) rend à peu près impossible une législation en ce sens mais aussi la résistance des employeurs en limite considérablement l'implantation dans les milieux de travail. De plus les syndicats eux-mêmes doutent de leur compatibilité avec le régime actuel de la négociation collective et les associent parfois aux comités créés dans les années 1920 dans le cadre du plan de représentation ouvrière (Employee Representation Plan) utilisés souvent par les employeurs pour éviter l'implantation d'un syndicat. En Angleterre la situation est plus favorable. D'abord les syndicats ne s'y opposent pas pourvu que l'on établisse la primauté de l'institution syndicale comme mécanisme de représentation. Cette position s'explique en grande partie par la plus grande facilité des syndicats anglais de les utiliser par rapport à leurs homologues américains comme des compléments à leur action. Ensuite les employeurs sont beaucoup moins réfractaires à leur présence qu'aux États-Unis. Malgré ces facteurs plus favorables, leur diffusion éventuelle demeure néanmoins restreinte.

La situation n'est guère plus encourageante dans le cas du partenariat patronalsyndical. L'auteur fait d'abord observer que les expériences de partenariat sont peu nombreuses dans les deux pays principalcment parce qu'elles sont implantées de façon volontaire par les parties sans aucune incitation légale spécifique. De plus, comme il prône la parité au sein des mécanismes de partenariat pour assurer la représentation indépendante des intérêts des salariés, il est obligé de constater que de tels cas sont très rares aux 
États-Unis et pratiquement inexistants en Angleterre. Deux études de cas l'amènent à conclure que pour réussir, le partenariat doit absolument correspondre aux trois exigences suivantes: être paritaire, être renforcé par la négociation collective et, finalement, produire des résultats tangibles pour les salariés dont la sécurité d'emploi. Ces conditions n'étant pas faciles à réunir, il est plutôt illusoire de miser sur le partenariat pour pallier la crise de la représentation.

La crise de la représentation ne peut donc être résorbée de façon significative par ces deux mécanismes supplémentaires. Est-il possible alors de croire dans la relance de l'institution syndicale pour rétablir son rôle-clé joué historiquement dans chaque système de relations industrielles? La conclusion identifie quelques facteurs favorables. L'auteur met en garde toutefois contre une vision trop optimiste, surtout parce que les politiques publiques ne semblent pas poursuivre cet objectif. L'auteur déplore la situation et termine en soulignant l'importance du syndicalisme comme mécanisme de représentation indépendante des intérêts des salariés. Le recul de la démocratie industrielle est peut-être l'indice de la diminution générale progressive du caractère démocratique de chacune des deux sociétés.

Cet ouvrage a pour but de plaider le libre accès des salariés à la représentation indépendante de leurs intérêts. Il a forcément un caractère normatif (que l'auteur reconnaît d'ailleurs) concentré sur l'institution syndicale. La thèse qui est très bien présentée avec moult détails sur chacun des deux pays souffre légèrement des défauts de sa qualité. À trop vouloir privilégier la représentation indépendante des intérêts des salariés et par conséquent l'organisation syndicale, le lecteur a parfois l'impression, surtout dans le chapitre huit et la conclusion, d'assister à une analyse subordonnée des deux autres mécanismes de représentation. Le lecteur veut bien reconnaître que l'organisation syndicale est le meilleur mécanisme institutionnalisé pour assurer la défense indépendante des intérêts des salariês mais la lecture de cet ouvrage ne le convainc pas que la représentation indépendante représente aujourd'hui ni le seul, ni, dans tous les cas, le meilleur moyen de défendre les intérêts des salariés. Malgré cet irritant, cet ouvrage qui, au demeurant est très bien fait, devrait intéresser tous ceux qui s'interrogent sur l'avenir du syndicalisme. Comme la situation a attiré récemment l'attention de nombreux spécialistes dans les deux pays et notamment aux États-Unis à l'occasion des travaux de la Commission Dunlop, l'ouvrage sera surtout apprécié par les Nord-Américains qui veulent connaître la situation anglaise et par les Anglais qui veulent connaitre celle des États-Unis. Le lecteur régulier de la revue Relations industrielles/Industrial Relations y trouvera un intérêt supplémentaire dans les nombreuses références à la situation canadienne.

MICHEL BROSSARD

Université de Montréal

\section{Labour Market Regimes and Patterns of Flexibility: A Sweden-Canada Comparison}

by Axel VAN DEN BERG, Bengt FURÅKER and Leif JOHANSSON, Lund: Arkiv, 1997, 264 p., ISBN 91-792-4099-2.

The basic question posed in his book by the authors is "does labour market security raise or lower workers' adaptability to various forms of industrial change?" (p. 25). The authors' attempt to understand this issue is framed within the context of a Canada-Sweden comparison and makes use of a variety of data sources such as labour force surveys, plant-level interviews of employees in 